\title{
Caracterización de sistemas ganaderos en seis municipios de Rivas y Carazo, Nicaragua
}

\section{Characterization of livestock systems in six municipalities in the regions of Rivas and Carazo, Nicaragua}

\section{Camilo Gutiérrez ${ }^{1}$, Bryan Mendieta ${ }^{2}$}

${ }^{1}$ Maestro en ciencia en producción animal. Instituto Nicaragüense de Tecnología Agropecuaria (INTA), ORCID: https://orcid.org/0000-0001-58568940/ ${ }^{2} \mathrm{PhD}$. En ciencia animal, ORCID: https://orcid.org/0000-0002-8077-7420, Universidad Nacional Agraria, Facultad de Ciencia Animal

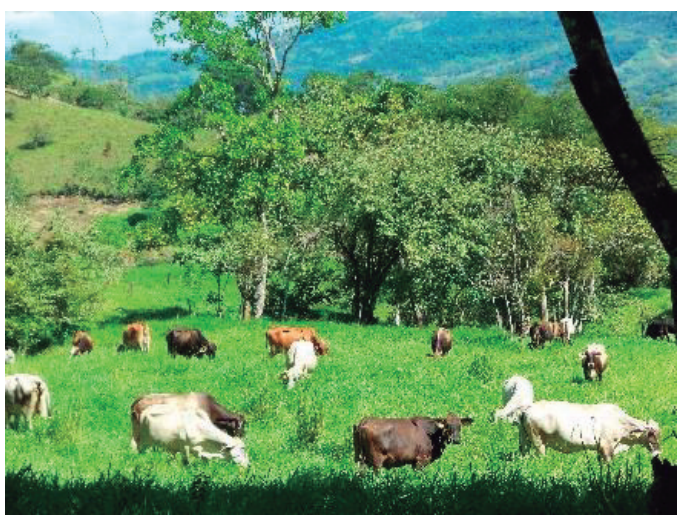

\section{RESUMEN}

Nicaragua es un país con características agropecuarias, destacando la producción ganadera. Se estudiaron 153 sistemas ganaderos de doble propósito en los departamentos de Rivas (Belén, Rivas y Tola) y Carazo (Santa Teresa, Diriamba y La Conquista). Se evaluó las características del sistema (áreas y distribución del sistema, tenencia de la propiedad y disponibilidad de agua), características del hato (raza, número de animales, mortalidad entre otros), características alimenticias (tipo de alimentación), manejo sanitario e infraestructura productiva. El objetivo de este estudio fue caracterizar sistemas ganaderos en los departamentos de Carazo y Rivas, Nicaragua. Se encontró que predominaron los propietarios, mayores a 50 años, el nivel de educación que prevalece es primaria, seguido de secundaria. Tamaño de fincas en un rango entre las 20 y 50 hectáreas. Durante el año hay momentos con alta producción de pasturas principalmente en la época lluviosa accesible al ganado, sin embargo, en la época seca existe escasez por lo que se debe suministrar minerales para solventar las demandas nutricionales. Las razas predominantes en el hato son: Pardo suizo y Brahman. La mortalidad anual en las crías y adultas fue baja inferior al $10 \%$. Las especies de pasto dominantes corresponden a Hyparrhenia rufa Nees (38.11\%), Andropogon gayanus kunth $(23.42 \%)$. La presencia de corrales y galeras constituyen las principales infraestructuras productivas. El manejo epizootiológico se desparasita, vitamina y vacuna el ganado contra las principales enfermedades presentes en los departamentos bajo estudio, siendo las afectaciones por arañas las más comunes, seguido de la presencia de parásitos internos y externo. Se identificaron la existencia de productores (28 de 153) que disponen de áreas con bancos forrajeros establecidos y algunas prácticas agroecológicas que favorecen la productividad.

Palabras clave: caracterización, sistemas ganaderos. pasto, Carazo, Rivas, escolaridad, hato ganadero.

\section{ABSTRACT}

Nicaragua is a country with agricultural characteristics, highlighting livestock production. We studied 153 double-purpose livestock systems in the departments of Rivas (Belén, Rivas and Tola) and Carazo (Santa Teresa, Diriamba and La Conquista). The characteristics of the system were evaluated (areas and distribution of the system, ownership of the property and availability of water), Characteristics of the herd (Race, Number of animals, mortality among others), nutritional characteristics (type of feeding), sanitary management and infrastructure productive The objective of this study was to characterize livestock systems in the departments of Carazo and Rivas, Nicaragua. It was found that the owners predominated, over 50 years old, the level of education that prevails is primary, and followed by secondary education. With average areas between 20 and 50 hectares. During the year there are times with high production of pastures mainly in the rainy season accessible to livestock, however in the dry season there is scarcity so that minerals must be supplied to meet the nutritional demands. In the herd they predominated: Swiss Brown and Brahman. Annual mortality in offspring and adults was lower than $10 \%$. The dominant grass species correspond to Hyparrhenia rufa Nees (38.11\%), Andropogon gayanus kunth $(23.42 \%)$. The presence of corrals and galleys constitute the main productive infrastructures. The epizootiological management is dewormed, vitamin and cattle vaccine against the main diseases present in the departments under study, being the affectations by spiders the most common, followed by the presence of internal and external parasites. We identified the existence of producers ( 28 of 153 ) that have areas with established forage banks and some agroecological practices that favor productivity.

Keywords: Characterization, cattle systems, pasture, Carazo, Rivas, schooling, cattle herd.
Recibido: 2 de febrero del 2018 Aceptado: 28 de mayo del 2018

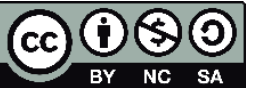

Los artículos de la revista La Calera de la Universidad Nacional Agraria, Nicaragua, se comparten bajo términos de la licencia Creative Commons: Reconocimiento, No Comercial, Compartir Igual. Las autorizaciones adicionales a las aquí delimitadas se pueden obtener en el correo freddy.aleman@ci.una.edu.ni

C) Copyright 2018. Universidad Nacional Agraria 


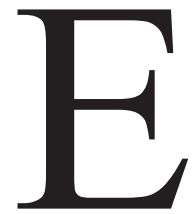

1 sector agropecuario es uno de los pilares fundamentales para la economía de Nicaragua. También, realiza aportes significativos a la generación de empleos y la seguridad alimentaria. El sector ganadero brinda aporte al sector económico, social y ambiental. Chuncho (2011) indica que la ganadería en Nicaragua, es heterogénea tanto en organización técnica, económica y de producción, así mismo hace mención que el nivel organizacional difiere de un sistema ganadero a otro, predominando el modelo de ganadería tradicional, en donde la alimentación del ganado se sustenta en las pasturas naturales que crecen de forma espontánea. Estradas y Holmann (2008) hacen mención que los sistemas ganaderos tradicionales se basan en el aprovechamiento de la vegetación herbácea espontánea y que la siembra de pastura son inferiores al 10\%. Holguín et al., (2003), en estudios realizados en Costa Rica, expresan que los sistemas ganaderos se sustentan en la alimentación de los animales mediante pasturas naturales de baja calidad y bajos rendimientos, en donde la productividad es limitada por la escases de forraje durante la época seca, lo que conlleva a bajos niveles de productividad de leche y carne.

En Nicaragua predominan los sistemas ganaderos tradicionales los cuales son extensivos y de doble propósito (CENAGRO, 2011). Vilaboa et al., (2009), hacen referencia que el sistema de doble propósito persigue dos objetivos principales la producción de leche, obtenida de manera manual, con el apoyo de becerro para estimular el descenso y la producción de carne mediante la cría de becerro y adultos que son descartado del sistema y pasan al suministro de carne del mercado. Gallo (2010) reporta que la ganadería tradicional es la responsable de la reducción de los bosques naturales vírgenes para ser transformados en fincas ganaderas. Iglesias et al., (2011), manifiestan que la actividad ganadera tradicional, está afectando los recursos naturales a un ritmo acelerado, por lo que se debe implementar estrategias silvopastoriles, para disminuir estas afectaciones en el ambiente. Monzote (2007) expresa que el cambio de los sistemas de ganadería tradicional a los sistemas ganaderos agroecológicos es cada vez más necesario y urgentes, ya que la ganadería agroecológica posee como principales características la diversidad espacial y temporal de forraje, autosuficiencia alimentaria y sobre todo la integración de la producción animal y vegetal, combinada con los aspecto socioeconómicos y ambientales.

Altieri y Nicholls (2002), mencionan que, dentro de los sistemas agropecuarios, se debe entender los aspectos sociales y las prácticas que el productor está im- plementando en su finca, lo que está relacionado directamente con el manejo y la búsqueda de sostenibilidad siendo la diversificación de cultivos y la conservación de los bosques y el recurso hídrico los principales parámetros para alcanzar la sostenibilidad. En este sentido, la caracterización de los sistemas ganaderos se hace necesario para el desarrollo de estrategias productivas para conocer los aspectos negativos de la ganadería tradicional e impulsar la ganadería agroecológica. El presente estudio se efectuó con el objetivo de caracterizar sistemas ganaderos en los departamentos de Carazo y Rivas, Nicaragua.

\section{MATERIALES Y MÉTODOS}

La metodología se desarrolló en seis etapas: inició con la identificación de los municipios a involucrar en el estudio; la determinación de la muestra (sistemas ganaderos a estudiar); elaboración, validación, llenado y control de calidad de la encuesta, procesamiento, análisis y generación de resultados y redacción de artículo. El presente estudio se realizó en 153 sistemas ganaderos de doble propósito (Producción de leche y carne), en dos departamentos y seis municipios, Carazo (Santa Teresa $(n=5)$, Diriamba $(n=16)$, La Conquista $(n=33)$ y el departamento de Rivas (Tola $(n=41)$, Belén $(n=31)$ y Rivas $(n=27)$, Nicaragua.

La cantidad de sistemas ganaderos por municipio se seleccionó, con base al IV censo Nacional Agropecuario de Nicaragua, identificando el número total de sistemas ganaderos existentes en los seis municipios bajo estudio.

Se realizó el cálculo de la muestra dividiendo el número de sistemas ganaderos por municipio, entre número de sistemas ganaderos totales en los seis municipios para determinar la proporción por municipio. Se diseñó una encuesta para recopilar información de los sistemas ganaderos con los siguientes aspectos.

Datos del productor: edad, sexo, nivel de escolaridad; características generales del sistema ganadero: área total, tenencia de la tierra, área de pasto, área de bosque, área de tacotal, fuentes hídricas (pozo, rio, quebradas y reservorios), financiamiento y nivel organizacional; característica del hato: raza, número de vacas paridas, número de vacas secas, número de novillos, número de terneros, número de toros, número de vaquillas, número de reproductores relación vaca-toro, mortalidad adultos y terneros, total de animales e introducción de vientre reproductivos; características alimenticias: tipo de alimentación ofrecida a los animales (sal común, melaza, concentrados, minerales, pastura, forraje); características productivas y reproductivas del hato: número de vacas en ordeño en invierno, número de vacas en ordeño en verano, producción de leche por vaca diaria, edad de destete y total de ingresos; 
manejo sanitario del ganado: vacunación, desparasitantes, vitamina, calendario fitosanitario, enfermedades comunes; infraestructura productiva en los sistemas ganaderos: corral, galera, picadora.
Las variables de interés para el estudio se describen en el cuadro 1 .

Cuadro 1. Listado de variables de interés en el estudio de caracterización de sistemas ganaderos en los departamentos de Carazo y Rivas, Nicaragua

\begin{tabular}{lc}
\hline \multicolumn{1}{c}{ Variables cuantitativas } & Unidad de clasificación \\
\hline $\begin{array}{l}\text { Área total de la finca: refiriéndose al área total del sistema ganadero } \\
\text { medida en hectárea, basándose en los datos obtenidos en la encuesta }\end{array}$ & Hectárea (ha) \\
Área de pasto: cantidad de área superficial con pastura & \\
Área de bosque: se refiere al área con cobertura boscosa & \\
Área de tacotal: se refiere al área con tacotal & \\
\hline Número de vacas paridas & \\
Número de vacas secas & \\
Número de novillos & Cantidad de animales \\
Número de terneros & \\
Número de toros & \\
Número de vaquillas & \\
Número de reproductores & \\
Mortalidad de adultos & \\
Mortalidad de terneros & \\
Cantidad de animales bovinos & \\
Introducción de vientres reproductivos & Dos categorías: Propio y alquilado \\
Número de vacas en ordeño en invierno & litros ${ }^{-1}$ vaca-1 día ${ }^{-1}$ \\
Número de vacas en ordeño en verano & Meses \\
\hline Producción de leche por día/vaca & Variables cualitativas \\
\hline Edad de destete & \\
\hline Total de ingreso & \\
\hline Tenencia de la tierra: se refiere a la situación legal del sistema & \\
ganadero & \\
\hline
\end{tabular}

Cuenta con pozo: refiriéndose si dentro del sistema se encuentra

un pozo de abastecimiento de agua para los animales

Presencia de rio en el sistema

Presencia de quebrada en el sistema

Dos categorías: Si y No

Recibe financiamiento

Si está organizado

\begin{tabular}{lc}
\hline Razas de ganado: Presencia de diferentes razas de ganado & Tipos de raza \\
\hline Sal común & \\
Melaza & \\
Concentrados & \\
Minerales & Dos categorías: Si y No \\
Pastura & \\
Forrajes & \\
Vacuna & \\
Vitamina & \\
Desparasita & Frecuencia de afectación \\
\hline Cuenta con calendario sanitario & \\
\hline
\end{tabular}


Análisis estadístico. La información que se generó en la caracterización se procesó con el programa informático SPSS (IBM Corp., 2010), generando las estadísticas descriptivas (promedio, desviación estándar, frecuencias, porcentajes, valores máximos y mínimos) para cada variable estudiada. Así mismo se realizó análisis mediante tablas de contingencias empleando prueba de chi-cuadrado y coeficiente de contingencia de cramer. El software empleado fue Infostat 2010 (Di Rienzo et ál., 2008).

\section{RESULTADOS Y DISCUSIÓN}

Aspectos sociales en los sistemas ganaderos. La población de los municipios estudiados, fueron agrupadas en cuatro categorías según la edad del productor o productora: el 15.75\% estuvo representada por adultos menores a los 40 años, $12.73 \%$ correspondieron a adultos entre los 40 y 44 años, la tercer categoría de edad se conformó con aquellos individuos mayores a 45 años y menores a 50 años encontrándose el $13.3 \%$ y la cuarta categoría agrupa a los adultos mayores de 50 años $59.39 \%$, esto indica que los sistemas ganaderos están siendo manejados en su mayoría por personas mayores de 50 años. Al relacionar la edad con el nivel de escolaridad se determinó que predomina la educación primaria $56.86 \%$ $(\mathrm{n}=87)$, seguido por la educación secundaria o bachillerato con un $22.22 \%(n=34)$, es notorio encontrar personas analfabetas $14.38 \%(\mathrm{n}=22)$, en su mayoría personas del sexo masculino. Las personas con educación técnica y superior son reducidas (cuadro 2). El Ministerio de Educación Cultura y Deporte (2004), hace mención que la educación es un pilar primordial y estratégico indispensable para el desarrollo social y económico de Nicaragua. El porcentaje de analfabetismo en la muestra de productores de este estudio fue de 14.47. Benavides y Morán (2013), reportan que en personas mayores a los 15 años y menores a los 64 años se presentan promedios de analfabetismos del $10.65 \%$ principalmente en los municipios más alejados de la capital.

La edad de los integrantes de la familia influye en muchos factores y toma de decisiones. Es posible que los ganaderos de mayor edad tengan más experiencia, recursos o autoridad que influya en las decisiones referentes al manejo de la finca, esta puede ser una desventaja debido al desempeño laboral que estos puedan tener. Posiblemente hay dos factores que inciden en el bajo porcentaje de personas entre los 20 y 40 años: uno es la falta de incentivos o atractivo económico para quedarse en las fincas y otro, asociado también al anterior, es la alta migración hacia otras ciudades del país y fuera del país. Para determinar la edad de los miembros del sistema se realizó un censo a cada hogar. En los municipios evaluados el mayor porcentaje de pobladores se encuentran en edades de 40 a 64 años para ambos sexos (figura 1).

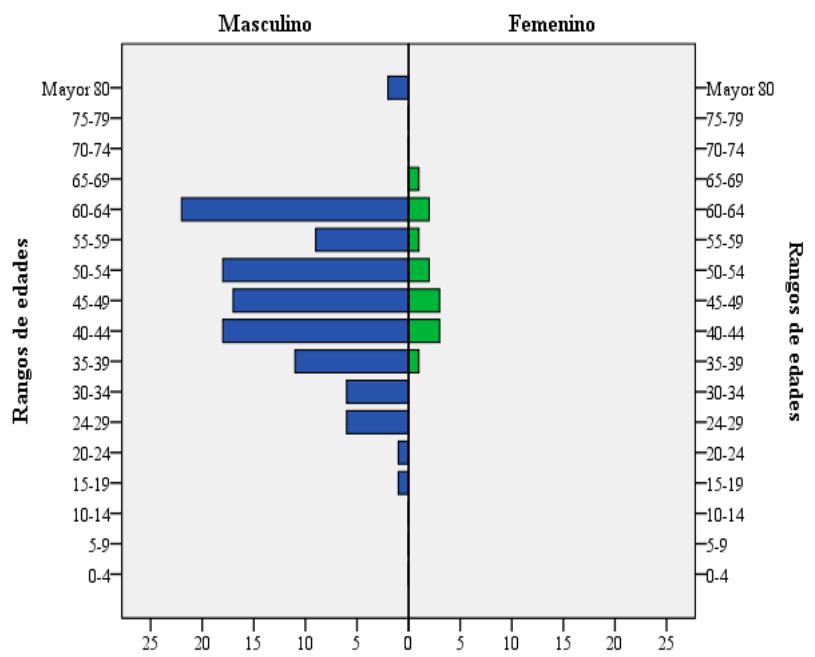

Figura 1. Pirámide poblacional según el sexo en los sistemas ganaderos en los municipios de Rivas y Carazo.
Cuadro 2. Distribución de rangos de edades por municipio y nivel de escolaridad de los propietarios en los sistemas ganaderos en los departamentos de Carazo y Rivas $(n=153)$

\begin{tabular}{lcccccccr}
\hline \multirow{2}{*}{ Municipios } & $15-19$ & $20-24$ & $25-29$ & $30-34$ & $35-39$ & $40-44$ & $45-50$ & $>50$ \\
\cline { 2 - 8 } & \multicolumn{7}{c}{ Porcentajes (\%) } \\
\hline Belén (n=31) & 0.65 & 0.65 & 0.65 & 0.65 & 0.65 & 3.27 & 2.61 & 11.11 \\
Diriamba (n=16) & 0.00 & 0.00 & 0.65 & 0.65 & 0.65 & 1.31 & 1.31 & 5.88 \\
La Conquista (n=42) & 0.00 & 0.00 & 1.31 & 1.31 & 3.27 & 3.27 & 1.31 & 16.99 \\
Rivas (n=27) & 0.00 & 0.00 & 0.00 & 0.00 & 1.96 & 1.31 & 2.61 & 11.76 \\
Santa Teresa (n=7) & 0.00 & 0.00 & 0.65 & 0.00 & 0.00 & 1.96 & 0.65 & 1.31 \\
Tola (42) & 0.00 & 0.00 & 0.65 & 1.31 & 1.31 & 2.61 & 4.57 & 16.99 \\
$\quad$ Escolaridad & & & & & & & & \\
Analfabeta & 0.00 & 0.65 & 0.00 & 1.31 & 0.65 & 1.96 & 2.61 & 7.19 \\
Alfabeta & 0.00 & 0.00 & 0.00 & 0.00 & 0.65 & 0.65 & 0.65 & 7.19 \\
Primaria & 0.65 & 0.00 & 1.31 & 2.61 & 2.61 & 7.19 & 5.88 & 36.60 \\
Secundaria & 0.00 & 0.00 & 2.61 & 0.00 & 0.65 & 2.61 & 3.92 & 12.42 \\
Educación técnica & 0.00 & 0.00 & 0.00 & 0.65 & 0.65 & 0.65 & 0.00 & 0.00 \\
Universidad & 0.00 & 0.00 & 0.00 & 0.00 & 2.61 & 0.65 & 0.00 & 0.00 \\
\hline
\end{tabular}

Características generales del sistema ganadero. El 83.03\% de los encuestados son propietario de sus sistemas ganaderos, el restante porcentaje rentan (alquilan) estos terrenos para dedicarse a la cría de ganado. También, es necesario destacar que la ganadería sigue siendo un empleo mayoritariamente masculino $(89.09 \%)$ con una participación del $10.91 \%$ de mujeres. En la distribución de la tierra para la producción bovina (cuadro 3) se evidencia 
que existen áreas superiores a las 200 hectáreas que practican la ganadería extensiva y sistemas que sobrepasan una hectárea dedicada a esta actividad económica. En el municipio de Belén las fincas ganaderas presentaron extensiones promedio de 41.51 hectáreas, seguida de Tola con 39.95 hectárea y Rivas con 30.92. En cambio, en el departamento de Carazo las mayores áreas promedios se reportaron en el municipio de Santa Teresa con promedios de 36.07 hectáreas de extensión, seguidas de La Conquista con 27.34 hectáreas y Diriamba con 24.50 hectáreas. De acuerdo a Chuncho (2011), en Rio Blanco y Matigüas, Nicaragua las áreas promedio están en el rango de 14.10 a 246 hectáreas dedicadas a la ganadería predominando el modelo extensivo en donde se dedican grandes extensiones de terreno para la cría de ganado, lo que concuerda con los resultados del presente estudio predominando este modelo ganadero.

En cuanto a la pastura predominan los pastos naturales (áreas donde no ha sembrado pastos en los últimos 15-20 años, en las que predominan especies naturales o joradas mientras que un $86 \%$ del área esta con pasturas nativas o naturalizadas de muy baja productividad y calidad, en los municipios evaluados el área con pasturas naturales no supera el $42 \%$ de los sistemas ganaderos siendo altamente significativos $(<0.0001)$ tanto en los municipios como en los sistemas ganaderos, encontrando las mayores áreas en los sistemas tradicionales. Polvorosa y Bastiaensen (2016) al analizar la ganadería en Nicaragua, la asocian con la deforestación esto debido al manejo extensivo de los animales.

En el presente estudio se encontró diferencias en cuanto a la cobertura boscosa y de tacotal en los municipios y sistemas ganaderos, siendo Belén el municipio con mayor presencia de bosque $(85.40 \%)$ y tacotales $(92.01 \%)$, los cuales son parte fundamental en el funcionamiento del sistema. Al consultar a los encuestados sobre su percepción sobre el estado de sus suelos el 37\% consideran que sus suelos son fértiles y el $65 \%$ consideran que sus suelos están degradados debido a la poca presencia de árboles dispersos en los potreros (cuadro 3).

Cuadro 3. Características generales de los sistemas ganaderos por municipios en Carazo y Rivas, Nicaragua

\begin{tabular}{|c|c|c|c|c|c|c|c|c|c|c|c|}
\hline \multirow{3}{*}{ Variables } & \multicolumn{2}{|c|}{ Tipo sist ganadero } & \multicolumn{6}{|c|}{ Municipios } & \multirow{2}{*}{\multicolumn{2}{|c|}{ Chi Cuadrado MV-G2 }} & \multirow{2}{*}{$\begin{array}{l}\text { Coef } \\
\text { Cont } \\
\text { Cram }\end{array}$} \\
\hline & Tra & Trad & Belén & Rivas & Tola & Conq & St Ter & Dir & & & \\
\hline & \multicolumn{8}{|c|}{$\%$ de Fincas } & Valor & $p$ & \\
\hline Área de la finca & 14.70 & 85.30 & 25.03 & 19.33 & 28.20 & 18.74 & 3.09 & 5.61 & 181.35 & 0.0001 & 0.16 \\
\hline Área de pasto de piso & 17.35 & 82.65 & 20.97 & 22.03 & 24.62 & 23.09 & 2.22 & 7.07 & 113.33 & 0.0001 & 0.20 \\
\hline Área de pasto de corte & 10.55 & 89.45 & 41.52 & 10.00 & 24.05 & 9.44 & 12.22 & 2.78 & 66.83 & 0.0001 & 0.51 \\
\hline Área de leguminosas & 14.14 & 85.86 & 28.27 & 7.07 & 53.35 & 0.00 & 11.31 & 0.00 & 2.28 & 0.5160 & 0.20 \\
\hline Área de tacotal & 7.99 & 92.01 & 56.87 & 16.11 & 8.49 & 14.41 & 0.25 & 3.87 & 52.00 & 0.0001 & 0.26 \\
\hline Área de bosque & 14.60 & 85.40 & 15.50 & 18.26 & 44.89 & 13.48 & 3.50 & 4.37 & 166.50 & 0.0001 & 0.29 \\
\hline
\end{tabular}

Tipo sist ganadero $=$ Tipo de sistema ganadero; Tra $=$ fincas en transición; Trad $=$ finca tradicional, Conq $=$ La Conquista, St Ter $=$ Santa Teresa, Dir $=$ Diriamba; Coef Cont Cram $=$ Coeficiente de contigencia Cramer.

naturalizadas como jaragua, grama, aceitillo, entre otras) principalmente en los sistemas ganaderos tradicionales (85.3\%), los mayores porcentajes se obtuvieron en los municipios de Belén (20.97\%), La Conquista (23.09\%) y Rivas $(22.03 \%)$ del área con presencia de este tipo de pastura. Vásquez (2006; citado por Chuncho, 2011) hace mención que en los municipios de Río Blanco y Paiwas, Nicaragua, se caracterizan por tener mayores extensiones de tierra disponibles para la producción ganadera, lo que significa mayor número de potreros para la actividad ganadera. Chuncho (2011) señala que en Nicaragua solo el $14 \%$ del área en pasturas se encuentra con gramíneas me-
Gallo (2010) en estudios realizados en Nicaragua expresa que el avance de la frontera agrícola principalmente para la implementación de la ganadería tradicional, pone en riesgo las fuentes hídricas. Se encontró que las principales fuentes hídricas de abastecimiento dentro de los sistemas ganaderos son: pozos $79 \%$, seguido de quebradas $63 \%$, rio $58 \%$ y en última instancia reservorios $4 \%$ (figura 2). Gallo (2010) asocia la perdida de recurso hídrico a la deforestación producto del incremento de la actividad ganadera. Gómez-Martínez et al., (2014), en estudios realizados en Matigüas, Nicaragua mencionan que los productores están más preocupados por la cantidad de agua 
disponible, sin preocuparse por la calidad de las misma, lo cual también aplica para los productores de los territorios incluidos en este estudio.

Características del hato. Los resultados evidencian que la estructura del hato está conformada principalmente por animales mestizos con diferentes cruces raciales, aclaran-

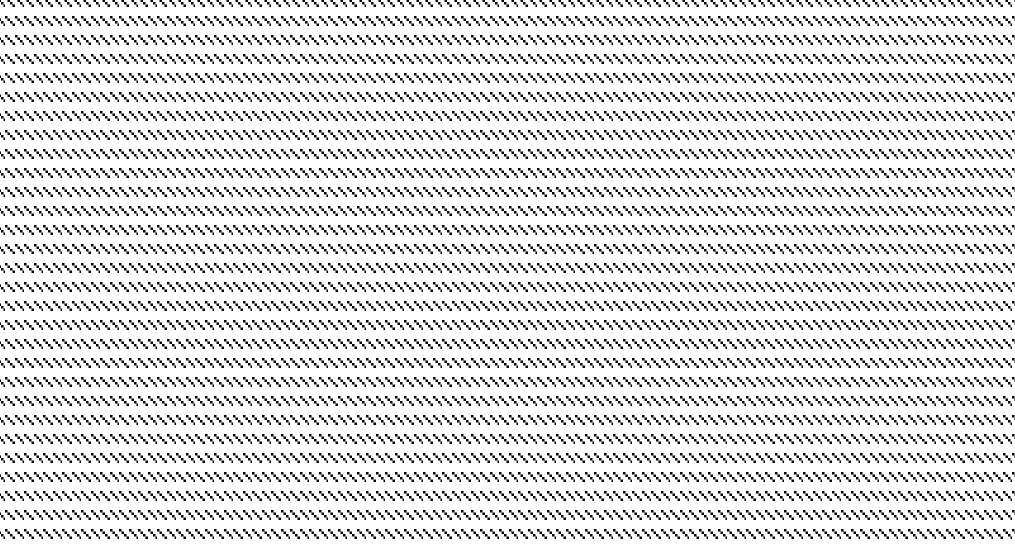

Figura 2. Abastecimiento de agua en sistemas ganaderos en Carazo y Rivas, Nicaragua.

do que en todos los sistemas no existen animales como razas puras, y se clasificaron como tipos raciales según la sangre que fenotípicamente predominaba. En sentido general, los productores prefieren los genotipos con mayor adaptación a las condiciones de manejo y alimentación característica de los sistemas tradicionales implementados en el trópico. Polvorosa y Basteaensen (2014), relacionan la mayor presencia de estos genotipos debido a las demandas de carne y leche por parte de la población nicaragüense. Así mismo Chuncho (2011), señala que en los municipios de Río Blanco y Paiwas, Nicaragua estos genotipos de ganado son los que predominan (figura 3).

La estructura de los hatos está conformada principalmente de hembras; predominan las vacas reproductoras, las hembras de dos años y las becerras menores de un año de edad, siendo altamente significativas tanto en los sistemas ganaderos como en los municipios $(<0.0001)$. La composición promedio de los hatos evidenció que en cada finca existen entre 2 y 42 vacas paridas, de las 3,789 cabezas de ganado existentes 829 son vacas paridas, lo que esta- blece una relación desde 2 a 12 vacas paridas por sistema ganadero, en los diferentes municipios. La incorporación de vientres de reemplazos al primer parto va por encima de los 24 meses de edad (como promedio), para las vaquillas (cuadro 4). De acuerdo a Chuncho (2011) la predominancia de animales hembras representa un potencial para el sistema ganadero ya que le confiere estabilidad económica al sistema en el tiempo al asegurar la presencia de vacas paridas (producción de leche) y la natalidad de becerros. Fajardo et al., (2014) mencionan que la cantidad de hembras reproductoras aptas son un parámetro fundamental en los sistemas ganaderos al producir leche y carne bajo el modelo de ganadería tradicional.

Características alimenticias. Estudio realizado por Chuncho (2011) en Río Blanco y Paiwas, Nicaragua indica la principal fuente de alimentación del ganado son las pasturas naturales, así mismo hace mención que la suplementación con sales minerales y concentrados es determinante principalmente en aquellos sistemas ganaderos dedicados a la producción de leche. El suministro de sal común, melaza y concentrados al ganado predomina en los sistemas ganaderos tradicionales, esto con la finalidad de suplir las demandas nutricionales por parte de los animales principalmente en la época seca. Iglesias et al., (2011) mencionan que una alimentación del ganado con forraje y suplementos alimenticios le confieren mayor resistencia a las enfermedades e incrementan su producción (cuadro 5).

Soto et al., (2008) en estudios realizados en Paiwas, Nicaragua menciona que las deficiencias nutricionales del ganado son solventadas por la incorpora-

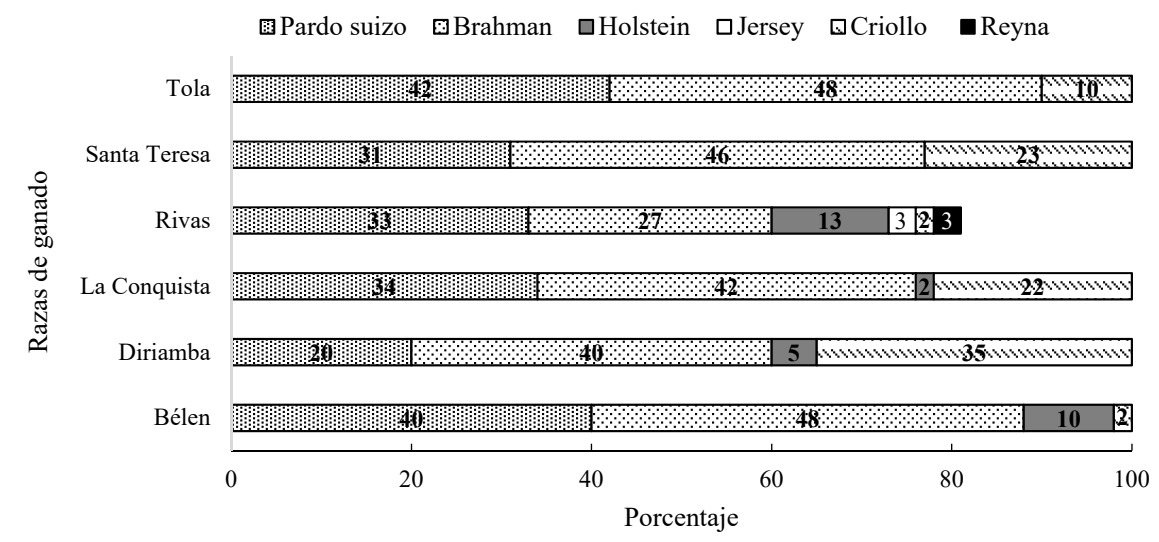

Figura 3. Principales genotipos de ganado encontrados en los sistemas ganaderos en los departamentos de Carazo y Rivas, Nicaragua. 


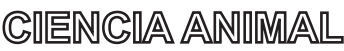

Cuadro 4. Composición del hato en los sistemas ganaderos en municipios de Carazo y Rivas, Nicaragua

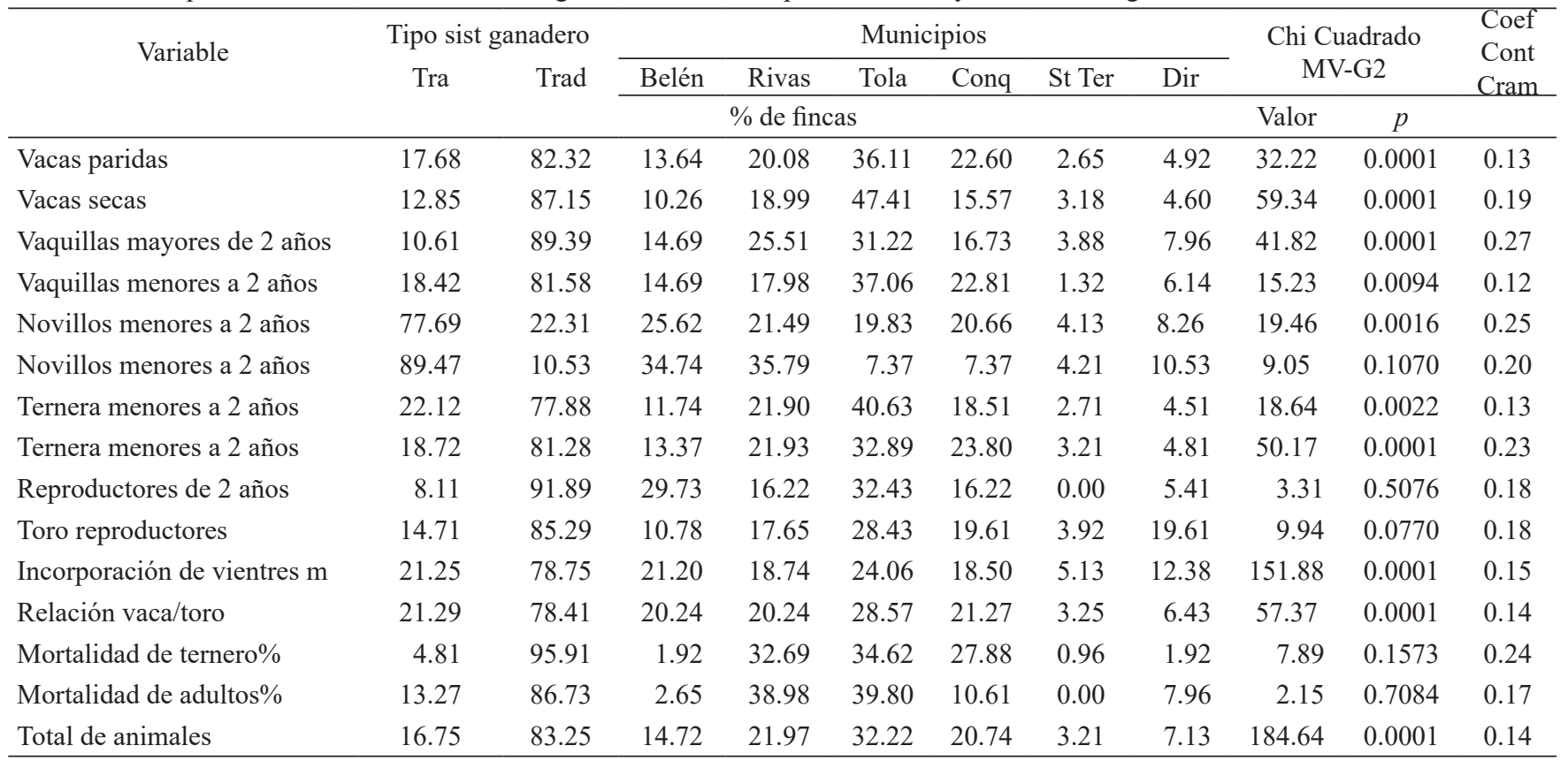

Tipo sist ganadero $=$ Tipo de sistema ganadero; Tra $=$ fincas en transición; Trad $=$ finca tradicional, Conq $=$ La Conquista, St Ter $=$ Santa Teresa, Dir $=$ Diriamba; Coef Cont Cram $=$ Coeficiente de contigencia Cramer.

ción de suplementos alimenticios para incrementar el consumo de forraje. Este mismo autor hace referencia que la deficiencia de energía son corregidos mediante el consumo de Saccharum officinarum (caña de azúcar), en el presente estudio el suministro de caña de azúcar persiste en los sistemas ganaderos tradicionales y en los municipios no superan el 20\% en términos generales, este mismo comportamiento es notorio al analizar el suministro de sal común, melaza ensilaje y minerales al hato.
$\mathrm{Al}$ analizar el área de pasto este se dividió en tres grandes componentes: pasto natural (todas aquellas pasturas naturales con altura menores a los $25 \mathrm{~cm}$ ), pasto de corte y leguminosas. El área de pasto de piso en los sistemas estudiados se constituyó de especies cultivadas. En la distribución de los pastos mejorados, predominaron el Hyparrhenia rufa Nees (38.11\%), Andropogon gayanus kunth $(23.42 \%)$, Dichantium aristatum Benth (15.12\%), Brachiaria Brizantha cv. Toledo y Mombaza (8.81\%),

Cuadro 5. Suplementación alimenticia proporcionada en los sistemas ganaderos en los departamentos de Carazo y Rivas, Nicaragua

\begin{tabular}{|c|c|c|c|c|c|c|c|c|c|c|c|}
\hline \multirow{3}{*}{ Variables } & \multicolumn{2}{|c|}{ Tipo de sit. ganadero } & \multicolumn{6}{|c|}{ Municipios } & \multirow{2}{*}{\multicolumn{2}{|c|}{$\begin{array}{c}\text { Chi Cuadrado } \\
\text { MV-G2 }\end{array}$}} & \multirow{3}{*}{$\begin{array}{l}\text { Coef. } \\
\text { Cont. } \\
\text { Cram }\end{array}$} \\
\hline & Tra & Trad & Bel & Riv & Tol & Conq & St Ter & Dir & & & \\
\hline & \multicolumn{8}{|c|}{$\%$ de Fincas } & Valor & $\mathrm{P}$ & \\
\hline Sal común & 18.39 & 81.69 & 20.69 & 17.24 & 27.01 & 20.69 & 2.87 & 11.49 & 9.64 & 0.0861 & 0.15 \\
\hline Melaza & 18.63 & 81.37 & 22.05 & 15.21 & 25.48 & 22.05 & 3.42 & 11.79 & 11.12 & 0.0491 & 0.13 \\
\hline Concentrados & 18.69 & 81.31 & 20.42 & 18.34 & 25.95 & 22.15 & 3.46 & 9.69 & 12.85 & 0.0248 & 0.13 \\
\hline Ensilaje & 18.31 & 81.69 & 20.00 & 17.63 & 26.44 & 22.03 & 3.05 & 10.85 & 10.46 & 0.0633 & 0.12 \\
\hline Guate & 19.44 & 80.56 & 18.33 & 18.33 & 28.89 & 21.11 & 3.33 & 10.00 & 8.77 & 0.1186 & 0.14 \\
\hline Caña de azúcar & 18.52 & 81.42 & 20.88 & 17.85 & 25.93 & 21.89 & 2.69 & 10.77 & 11.34 & 0.0451 & 0.12 \\
\hline Minerales ocasionales & 19.44 & 80.56 & 19.84 & 18.65 & 26.59 & 22.62 & 3.17 & 9.13 & 12.93 & 0.0241 & 0.14 \\
\hline Minerales inyectados & 19.57 & 80.43 & 21.00 & 16.73 & 27.05 & 22.06 & 3.20 & 9.96 & 11.58 & 0.0411 & 0.13 \\
\hline Minerales permanentes & 18.51 & 81.49 & 21.00 & 18.51 & 25.27 & 21.35 & 2.85 & 11.03 & 10.23 & 0.0690 & 0.12 \\
\hline
\end{tabular}

Tipo sist ganadero $=$ Tipo de sistema ganadero; Tra $=$ fincas en transición; Trad $=$ finca tradicional, Conq $=$ La Conquista, $\mathrm{St}$ Ter $=$ Santa Teresa, Dir $=$ Diriamba; Coef Cont Cram $=$ Coeficiente de contigencia Cramer. 
otras especies de pasto $(7.71 \%)$ y pasturas naturales (6.83\%). Al analizar los pastos de corte predomina Pennisetum sp en todos los sistemas evaluados (Cuadro 6). La presencia de las leguminosas, en los sistemas ganadero fue muy escasas predominando Gliricidia sepium y Guazuma ulmifolia, las cuales fueron establecidas por los ganaderos.
Cardenal (2005), menciona que el manejo que el productor implementa en sus pastizales, limitan el aprovechamiento óptimo de la capacidad forrajera de la especie cultivada. Holmann et al., (2004) señala, que en Nicaragua y Panamá la adopción de gramíneas es baja y se sitúa en un rango de 24 al 55\% en la producción de leche y 5 y $18 \%$ en carne.

Cuadro 6. Composición de la pastura en los sistemas ganaderos en los departamentos de Carazo y Rivas, Nicaragua

\begin{tabular}{|c|c|c|c|c|c|}
\hline \multicolumn{2}{|l|}{ Pastura } & \multicolumn{2}{|c|}{ Pasto de corte } & \multicolumn{2}{|c|}{ Leguminosas } \\
\hline Belén & Porcentaje (\%) & & Porcentaje (\%) & & Porcentaje (\%) \\
\hline Dichantium aristatum Benth & 9.27 & & 3.70 & Gliricidia sepium & 13.33 \\
\hline Pastura Natural & 2.44 & & 7.41 & & \\
\hline Andropogon gayanus kunth & 0.98 & & 7.41 & & \\
\hline Hyparrhenia rufa (Nees) & 4.88 & & 0.00 & & \\
\hline \multicolumn{6}{|l|}{ Rivas } \\
\hline Cynodon spp & 0.98 & & & Gliricidia sepium & 13.33 \\
\hline Brachiaria Brizantha cv. Toledo & & & 14.81 & & \\
\hline Andropogon gayanus kunth & 1.95 & & & Guazuma ulmifolia & 6.67 \\
\hline Panicum máximum cv. Mombaza & 0.98 & & & & \\
\hline Hyparrhenia rufa (Nees) & 9.27 & & & & \\
\hline Brachiaria Brizanta & 0.98 & & & & \\
\hline Dichantium aristatum Benth) & 2.44 & & & & \\
\hline \multicolumn{6}{|l|}{ Tola } \\
\hline Cynodon spp & 2.93 & Pennisetum sp & 14.81 & Gliricidia sepium & 13.33 \\
\hline Pastura Natural & 0.98 & Cynodon spp a & 7.41 & Guazuma ulmifolia & 13.33 \\
\hline Andropogon gayanus kunth & 4.39 & & & & \\
\hline Panicum máximum cv. Mombaza & 2.93 & & & & \\
\hline Hyparrhenia rufa (Nees) & 11.71 & & & & \\
\hline Brachiaria Brizanta & 2.44 & & & & \\
\hline Dichantium aristatum Benth) & 3.41 & & & & \\
\hline \multicolumn{6}{|l|}{ La conquista } \\
\hline Brachiaria Brizantha cv. Toledo & 0.49 & Pennisetum sp & 14.81 & Gliricidia sepium & 33.33 \\
\hline Pastura Natural & 1.46 & Brachiaria Brizantha & 18.52 & & \\
\hline Andropogon gayanus kunth & 8.78 & & & & \\
\hline Hyparrhenia rufa (Nees) & 8.78 & & & & \\
\hline Brachiaria Brizanta & 1.46 & & & & \\
\hline \multicolumn{6}{|l|}{ Santa Teresa } \\
\hline Andropogon gayanus kunth & 2.93 & Hyparrhenia rufa (Nees) & 3.70 & & \\
\hline Hyparrhenia rufa (Nees) & 2.93 & Pennisetum sp & 3.70 & & \\
\hline \multicolumn{6}{|l|}{ Diriamba } \\
\hline Andropogon gayanus kunth & 4.39 & Pennisetum sp & 3.70 & Gliricidia sepium & 6.67 \\
\hline Hyparrhenia rufa (Nees) & 0.98 & & & & \\
\hline Brachiaria Brizanta & 0.49 & & & & \\
\hline Panicum máximum cv. Mombaza & 0.49 & & & & \\
\hline Pastura Natural & 1.95 & & & & \\
\hline
\end{tabular}


Mediante el análisis de componentes principales, se determinó que los tres primeros componentes aislaron el $66 \%$ de la varianza entre los sistemas productivos y los municipios bajo estudio. Se observó una correlación negativa entre los sistemas ganaderos en transición y la utilización de melaza y urea, así como el alquiler de pasturas, esto se debe probablemente a que las áreas de pastos de estos sistemas satisfacen la demanda de forraje por parte del ganado, las fincas en transición se están implementando alternativas como caña de azúcar y minerales inyectados. Existe una relación positiva de los sistemas tradicionales y el suministro de concentrados, ensilaje y el uso de melaza (figura 4).

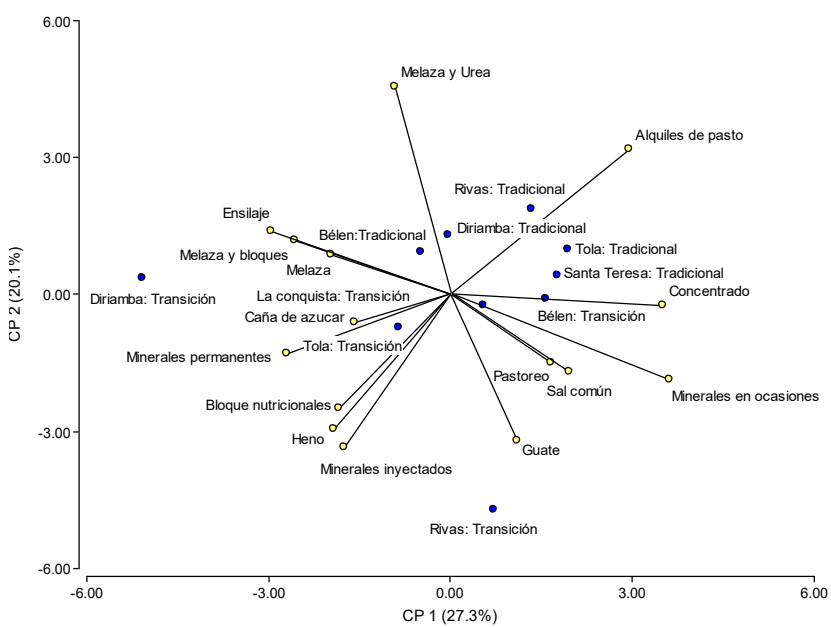

Figura 4. Localización en el espacio bidimensional de las variables de interés en las características de los sistemas ganaderos en los departamentos de Carazo y Rivas, Nicaragua. cional supera en un $7.92 \%$ a los sistemas en transición (cuadro 7).

Al analizar la época lluviosa es notorio encontrar $31.36 \%$ se encuentran produciendo entre uno y cuatro litros por vaca al día, sin embargo, el $73.84 \%$ producen entre cuatro y 10 litros al día, esto se debe a la disponibilidad de alimento principalmente pastura y agua. En cambio, en la época seca el $13.06 \%$ de las vacas no logra producir un litro de leche, el $71.89 \%$ produce entre uno y cuatro litros y el restante porcentaje llega a producir entre cuatro y 10 litros. Esto conlleva a disminuir la oferta de leche durante la época seca. Chuncho (2011) hace mención que para mantener la producción de leche se deben incorporar tecnologías en los sistemas

Cuadro 7. Comportamiento en la producción de leche en los sistemas ganaderos en los departamentos de Carazo y Rivas, Nicaragua

\begin{tabular}{|c|c|c|c|c|c|c|c|c|c|c|c|}
\hline \multirow[t]{3}{*}{ Variables } & \multicolumn{2}{|c|}{$\begin{array}{l}\text { Tipo de sist. } \\
\text { ganaderos }\end{array}$} & \multicolumn{6}{|c|}{ Municipios } & \multirow{2}{*}{\multicolumn{2}{|c|}{$\begin{array}{l}\text { Chi Cuadrado } \\
\text { MV-G2 }\end{array}$}} & \multirow{3}{*}{$\begin{array}{l}\text { Coef. } \\
\text { Cont. } \\
\text { Cram }\end{array}$} \\
\hline & Tra & Trad & Bel & Riv & Tol & Conq & St Ter & Dir & & & \\
\hline & \multicolumn{8}{|c|}{$\%$ de Fincas } & Valor & $\mathrm{P}$ & \\
\hline Vacas en ordeño en invierno & 25.55 & 74.45 & 14.73 & 30.77 & 24.90 & 19.42 & 1.98 & 8.21 & 278.06 & $<0.0001$ & 0.31 \\
\hline Vacas en ordeño en verano & 24.44 & 75.56 & 16.68 & 29.19 & 21.57 & 22.81 & 1.92 & 7.83 & 116.03 & $<0.0001$ & 0.29 \\
\hline Producción de leche & 18.73 & 81.27 & 22.08 & 19.04 & 25.85 & 20.27 & 3.21 & 9.56 & 56.47 & $<0.0001$ & 0.13 \\
\hline Total de ingreso & 46.04 & 53.96 & 8.38 & 59.19 & 29.59 & 2.25 & 0.27 & 0.32 & 412.85 & $<0.0001$ & 0.51 \\
\hline
\end{tabular}

Tipo sist ganadero $=$ Tipo de sistema ganadero; Tra $=$ fincas en transición; Trad $=$ finca tradicional, Conq $=$ La Conquista, St Ter $=$ Santa Teresa, Dir= Diriamba; Coef Cont Cram $=$ Coeficiente de contigencia Cramer.

Características reproductivas del hato. Magaña et al., (2006) hace mención que los indicadores de producción de leche en el trópico de América Latina, están entre 2,8 a 6,5 litros $^{-1} \mathrm{vaca}^{-1} \mathrm{dí}^{-1}$. En los municipios y sistemas ganaderos bajo estudio existe una diferencia significativa $(<0.0001)$, independientemente de la época ya sea seca o lluviosa, mostrando la mayor producción los sistemas tradicionales y el municipio de Rivas (cuadro 7). El MAGFOR (2008) señala que la producción promedio nicaragüense es de 3.5 litros $^{-1} \mathrm{vaca}^{-1} \mathrm{dí}^{-1}$, Torres (2007) en su estudio encontró producciones de 3.8 litros $^{-1} \mathrm{vaca}^{-1} \mathrm{dí}^{-1}$ en Matagalpa y Jinotega, Nicaragua. Al analizar las variables económicas los mayores ingresos se reportan en el municipio de Rivas, sin embargo, al comparar los sistemas ganaderos el tradi- ganaderos como son los sistemas silvopastoriles y el suministro de fuentes minerales al ganado y los bancos forrajeros (cuadro 8).

Manejo sanitario del ganado. En cuanto al manejo sanitario del ganado en todos los sistemas evaluados, en los diferentes municipios no fue significativa, menos del $30 \%$ realizan prácticas como es la vacunación, vitamina y desparasitan al ganado y cuentan con un calendario sanitario, principalmente en los sistemas tradicionales (cuadro 9). Chuncho (2011) se refiere que estas prácticas sanitarias son implementadas en los sistemas ganaderos de Paiwas y Rio blanco, Nicaragua para contrarrestar las afectaciones por enfermedades. 
Cuadro 8. Producción de leche en los sistemas ganaderos en los departamentos de Carazo y Rivas ( $\mathrm{n}=153)$

\begin{tabular}{|c|c|c|c|c|c|}
\hline \multirow{2}{*}{$\begin{array}{l}\text { Producción de leche } \\
\text { (Litros) }\end{array}$} & \multicolumn{5}{|c|}{ Municipios } \\
\hline & Bel & Dir Conq & Riv $S$ & St Ter & Tol \\
\hline $\begin{array}{l}\text { Invierno (promedio litro } \\
\text { vaca }^{-1} \text { día }^{-1} \text { ) }\end{array}$ & \multicolumn{5}{|c|}{ Porcentajes (\%) } \\
\hline$\overline{<1}$ & 0.00 & $\begin{array}{ll}0.00 & 0.00\end{array}$ & 0.00 & 0.00 & 0.00 \\
\hline $1-4$ & 5.88 & 0.6510 .46 & 3.92 & 0.65 & 9.80 \\
\hline $4-10$ & 13.72 & 9.1516 .99 & 13.07 & 3.92 & 16.99 \\
\hline $10-15$ & 0.65 & 0.650 .00 & 0.65 & 0.00 & 0.65 \\
\hline$\geq 15$ & 0.00 & $0.00 \quad 0.00$ & 0.00 & 0.00 & 0.00 \\
\hline \multicolumn{6}{|l|}{$\begin{array}{l}\text { Verano (promedio litro } \\
\text { vaca }^{-1} \text { día }^{-1} \text { ) }\end{array}$} \\
\hline$\overline{<1}$ & 1.96 & $0.65 \quad 3.92$ & 1.96 & 0.00 & 4.57 \\
\hline $1-4$ & 13.72 & 8.5018 .30 & 10.46 & 2.61 & 18.30 \\
\hline $4-10$ & 4.57 & $1.31 \quad 5.23$ & 5.23 & 1.96 & 4.57 \\
\hline $10-15$ & 0.00 & $0.00 \quad 0.00$ & 0.00 & 0.00 & 0.00 \\
\hline$>15$ & 0.00 & $0.00 \quad 0.00$ & 0.00 & 0.00 & 0.00 \\
\hline
\end{tabular}

Bel $=$ Bélen, Tol $=$ Tola, Conq $=$ La Conquista, St Ter $=$ Santa Teresa, Dir= Diriamba.

Principales enfermedades. En cuanto al estado sanitario del ganado en todos los sistemas evaluados, las mayores afectaciones están relacionadas al ataque de arañas o pododermatitis, que afecta la pezuña de la vaca, dificultando su movilidad para alimentarse. Seguido por los parásitos externos y enfermedades bacterianas. En términos generales el ganado no se encuentra en peligro ya que la mayoría de los productores expresaron que sus animales no presentan ninguna afectación provocada por microorganismos y virus. Livas (2014) reportó que la incidencia de enfermedades está relacionada con el manejo que el productor ejerce sobre el hato de manera directa (figura 5).

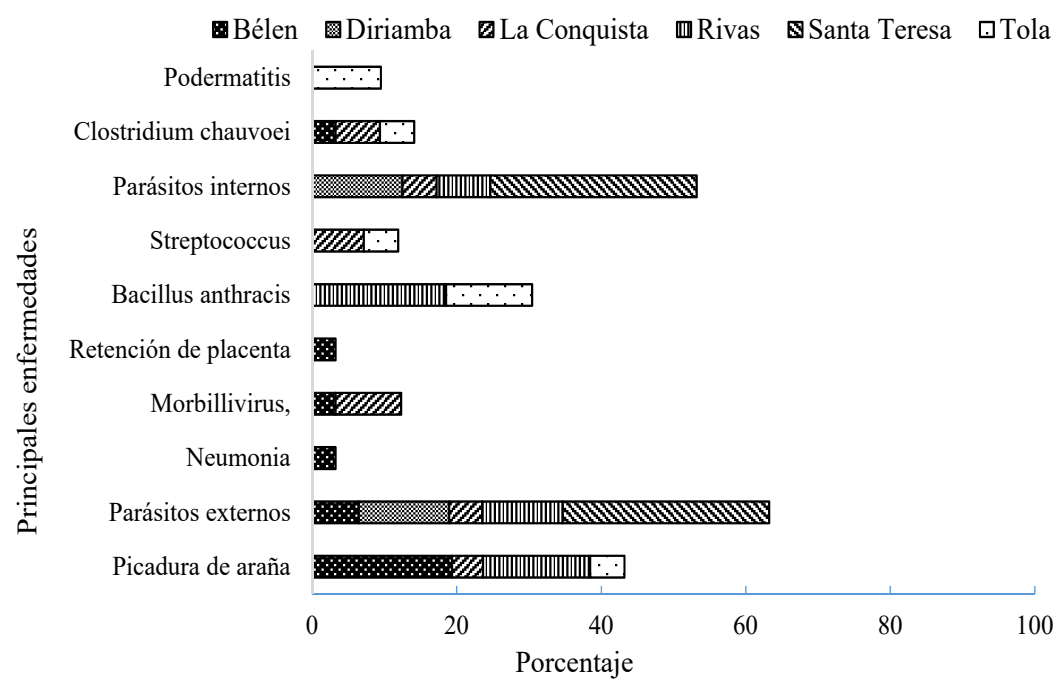

Figura 5. Principales enfermedades expresadas por los productores en los sistemas ganaderos en los departamentos de Carazo y Rivas, Nicaragua.
Infraestructura productiva en los sistemas ganaderos. La evaluación de la disponibilidad de equipamiento (Cuadro 5) indicó que en todos los municipios se cuentan con corrales para mantener el ganado. Sin embargo, la existencia de galeras, picadoras de pasto es mínima, esto representa una desventaja para el manejo del ganado en términos de sanidad y alimentación por parte del productor (figura 6). Castillo et al., (2014), en estudio realizado en Campeche, México expresan que la disponibilidad de infraestructura productiva representa un potencial para el desarrollo de la ganadería.

Presencia de especies forrajeras. Se determinó la presencia de especies forrajeras en los sistemas ganaderos, los bancos de proteína están presente en todos los municipios predominando en La Conquista $(50 \%$ de los sistemas ganaderos), seguido de Rivas. La presencia de Leucaena leucacephala, Guazuma ulmifolia, Moringa olifera, Gliricidia sepium, se encuentran en menos del $40 \%$ de los sistemas ganaderos, estas son establecidas principalmente como cercas vivas. De acuerdo a Villanueva et al., (2009), las presencias de bancos forrajeros ofrecen recursos alimenticios para la alimentación animal en la época seca cuando los pastos reducen su disponibilidad y calidad de la materia seca comestible. Con respecto a la existencia de estos recursos forrajeros en el sistema ganadero no está relacionado directamente al tipo de sistema.

López et al., (2007), manifiestan que, por la adición de leguminosas arbustivas, en las pasturas contribuyen a mejorar la alimentación del ganado e incrementar la producción de leche. La presencia de Cratylia argéntea es reducida, en los sistemas ganaderos por lo que se debe promover estas tecnologías en el manejo de la alimentación de ganado en la época seca (Figura 7). Castillo et al., (2014), en estudio realizado en Campeche, México hace referencia que se deben introducir y promover alternativas alimenticias en los sistemas ganaderos, principalmente para solventar las deficiencias nutricionales del ganado en época seca. Chuncho (2011) en su estudio en Rio Blanco y Paiwas, Nicaragua que la combinación de gramíneas (caña de azúcar y king grass verde) más leguminosas arbustivas (Cratilya argentea y Gliricidia sepium) son una alternativa ante la limitante de forraje. Sepúlveda (2008; citado por chuncho, 2011) en es- 
Cuadro 9. Principales prácticas sanitarias implementadas en los sistemas ganaderos en los departamentos de Carazo y Rivas, Nicaragua

\begin{tabular}{|c|c|c|c|c|c|c|c|c|c|c|c|}
\hline \multirow{3}{*}{ Variables } & \multicolumn{4}{|c|}{ Tipo de sist. ganaderos } & \multicolumn{4}{|c|}{ Municipios } & \multirow{2}{*}{\multicolumn{2}{|c|}{$\begin{array}{l}\text { Chi Cuadrado } \\
\text { MV-G2 }\end{array}$}} & \multirow{3}{*}{$\begin{array}{l}\text { Coef. } \\
\text { Cont. } \\
\text { Cram }\end{array}$} \\
\hline & Tra & Trad & Bel & Riv & Tol & Conq & St Ter & Dir & & & \\
\hline & & & \multicolumn{6}{|c|}{$\%$ de Fincas } & Valor & $\mathrm{P}$ & \\
\hline Cuenta con calendario sanitario & 18.97 & 81.03 & 19.76 & 17.79 & 26.88 & 21.34 & 3.95 & 10.28 & 9.44 & 0.0927 & 0.12 \\
\hline Vacunan & 17.86 & 82.14 & 20.83 & 17.86 & 28.57 & 20.24 & 2.98 & 9.52 & 5.90 & 0.3161 & 0.12 \\
\hline Vitamina & 18.35 & 81.65 & 20.89 & 17.72 & 26.58 & 21.52 & 3.16 & 10.13 & 6.53 & 0.2579 & 0.13 \\
\hline Desparasita & 18.99 & 81.01 & 20.89 & 17.72 & 27.22 & 20.89 & 3.16 & 10.13 & 6.19 & 0.2886 & 0.13 \\
\hline
\end{tabular}

Tipo sist ganadero $=$ Tipo de sistema ganadero; Tra $=$ fincas en transición; Trad $=$ finca tradicional, Conq $=$ La Conquista, St Ter $=$ Santa Teresa, Dir $=$ Diriamba; Coef Cont Cram $=$ Coeficiente de contigencia Cramer.

tudios realizados en Matigüas, Nicaragua reporto que el $75 \%$ de los productores emplean bancos forrajeros, se-

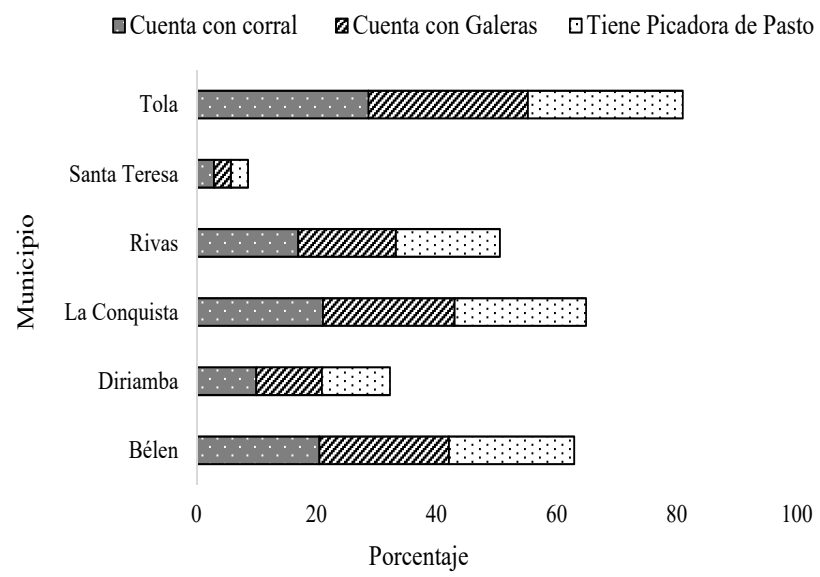

Figura 6. Disponibilidad de infraestructura productiva en los sistemas ganaderos en los departamentos de Carazo y Rivas, Nicaragua.

\section{CONCLUSIÓN}

En los sistemas ganaderos predominan los propietarios, mayores a 50 años, el nivel de educación que prevalece es primaria, seguido de secundaria. Predominan los propietarios, catalogados como medianos productos, con disponibilidad de pasto durante todo el año, prevaleciendo el cultivo de poaceae en los pastizales, se suministra suplementos minerales al ganado para compensar las demandas nutricionales. En el hato predominan los genotipos Brahaman y Pardo Suizo, con producción de leche por vaca similar al promedio nacional reduciéndose en la época seca guido de las pasturas mejoradas, así como la protección del bosque y las fuentes de agua.

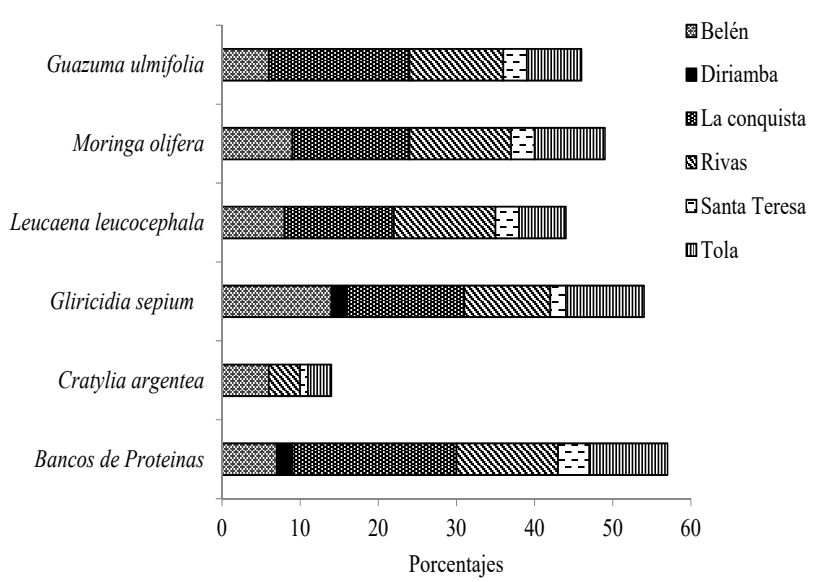

Figura 7. Presencia de Bancos de proteínas en los sistemas ganaderos en los departamentos de Carazo y Rivas, Nicaragua.

debido a la falta de forraje y agua. La mortalidad tanto en cría como en adulto fue baja inferior al 10\%. La edad de destete y el periodo entre parto es superior a los 36 meses. Los ganaderos realizan prácticas de vacunación, desparasitante y vitamina al hato ganadero. En cuanto a la estructura productiva prevalece el corral y en menor grado la galera y picadora de pasto. El manejo de las enfermedades es generalizado en todos los sistemas predominando las picaduras de araña y los parásitos internos y externos. Los bancos de proteínas son poco implementados en los diferentes municipios y sistemas ganaderos. 


\section{REFERENCIAS BIBLIOGRÁFICAS}

Altieri, M., y Nicholls, C. (2002). Un método agroecológico rápido para la evaluación de la sostenibilidad de cafetales in Manejo Integrado de Plagas y Agroecología (CATIE). (no.64) p. 17-24.

Benavides-González, Á., \& Morán-Centeno, J. C. 2013. Análisis numérico de características básicas de unidades familiares productivas (UFP) en nueve comunidades rurales de Nicaragua. La Calera, 13(21), 101-109.

Castillo, A. D., Sardiñas López, Y., Castillo Corría, E., Padilla Corrales, C., Jordán Vázquez, H., Martínez Zubiaur, R. O., \& Gómez Cruz, O. (2014). Caracterización de ranchos ganaderos de Campeche, México. Resultados de proyectos de transferencia de tecnologías. Avances en Investigacion Agropecuaria, 18(2)

Chuncho, M; C, G. (2011). Análisis de la percepción y medidas de adaptación al cambio climático que implementan en la época seca los productores de leche en Río Blanco y Paiwas, Nicaragua. (Doctoral dissertation).

Di Rienzo J.A., Casanoves F., Balzarini M.G., Gonzalez L., Tablada M., Robledo C.W. 2008. InfoStat, versión 2008, Grupo InfoStat, FCA, Universidad Nacional de Córdoba, Argentina.

Estrada, R y Holmann, F. 2008. Competitividad de los Pequeños Productores de Leche frente a los Tratados de Libre Comercio en Nicaragua, Costa Rica y Colombia. Centro Internacional d Agricultura Tropical (CIAT), International Livestock Research Institute (ILRI). Cali, Colombia. 70p. (Documento de Trabajo no. 207).

Fajardo, M. Y., Vargas, G. F., \& Marín, L. A. V. (2014). Costos ambientales y evaluación social en conversión de los Sistemas de producción ganadera tradicional al sistema silvopastoril en fincas ganaderas de los municipios de Florencia, Morelia y Belén de los Andaquíes del departamento del Caquetá. Momentos de Ciencia, 11 (1).

Gallo, J. (2010). Variables ambientales y actividades ganaderas determinantes para la Evaluación Ambiental Estratégica de la ganadería bovina en Nicaragua. Nexo Revista Cientifica, 23(1), 2-8.

Gómez-Martínez, M. J., Gutiérrez-Montes, I., \& Declerck, F. (2014). Percepción local acerca de la calidad del agua y la herpetofauna en fincas ganaderas del municipio de Matiguás, Nicaragua. Revista Luna Azul, (38).

Holguin, V.; Ibrahim, M.; Mora, J.; Rojas, A. 2003. Caracterización de sistemas de manejo nutricional en ganaderías de doble propósito de la región Pacífico Central de Costa Rica. Agrofor Am 10 (39/40): 40-46.

Holguin, V.; Ibrahim, M.; Mora, J.; Rojas, A. 2003. Caracterización de sistemas de manejo nutricional en ganaderías de doble propósito de la región pacifico central de Costa Rica. Agroforestería en las Américas V. 10 (39-40): 40-46.

Holmann F., Rivas, L.; Argel P. y Pérez, E. 2004. Impact of the adoption of Brachiaria grasses: Central America and Mexico. Livest. Res. Rural Devel. (en línea). Consultado el 10 de noviembre de 2017. Disponible en http://sian.inia.gob.ve/repositorio/revistas ci/ ZootecniaTropical/zt2603/pdf/andrade_h.pdf

IBM SPSS Statistics 19 Command, Syntax Reference. Copyright (C) SPSS Inc. 1989, 2010. IBM SPSS Statistics versión 19. 2483 p.

Iglesias, J. M., Funes-Monzote, F., Toral, O. C., Simón, L., \& Milera, M. (2011). Diseños agrosilvopastoriles en el contexto de desarrollo de una ganadería sustentable. Apuntes para el conocimiento. Pastos y forrajes, 34(3), 241-257.

IV Censo Nacional Agropecuario 2010-2011 (CENAGRO). (2013) Instituto Nacional de Desarrollo, INIDE. Ministerio Agropecuario y Forestal, MAGFOR.

Livas, C. F. (2014). Experiencias en producción de carne bovina bajo pastoreo en el trópico. Centro de enseñanza, investigación y extensión de ganadería tropical (CEIEGT), Universidad Nacional Autónoma de México, México, D.F. Disponible en: http://www. fmvz.unam.mx/fmvz/departamentos/rumiantes/ bovinotecnia/BtRgz 00g023.pdf (Consultada en octubre de 2017).

López, F.; Gómez, R.; Harvey, C.; López, M.; Sinclair, F. 2007. Toma de decisiones de productores ganaderos sobre el manejo de los árboles en potreros en Matiguás, Nicaragua. Avances de Investigación. Agroforestería en las Américas N ${ }^{\circ} 45$ 2007. Pp 93-100

Magaña, J.; Ríos, G.; Martínez, J. 2006. Los sistemas de doble propósito y los desafíos en los climas tropicales de México. Universidad Autónoma de Yucatán, Yucatán, México. Arch. Latinoam. Prod. Anim. 2006. Vol. 14 (3): 105-114 105 pp.

MAGFOR (Ministerio Agropecuario y Forestal de Nicaragua) 2008. Subprograma Inversión de desarrollo integral para la producción de leche en polvo de alcance regional. Managua, Nicaragua. $100 \mathrm{pp}$

MECD (Ministerio de educación, cultura y deportes, NI). 2004. El desarrollo de la educación. Informe Nacional de Nicaragua. Noviembre 201737 p. http://www.ibe.unesco.org/International/ICE47/English/Natreps/reports/nicaragua_part_1.pdf.

MONZOTE, F. F. (2007). De la especialización ganadera a la ganadería agroecológica: Experiencias cubanas en investigación y producción. Revista Brasileira de Agroecologia, 2(2).

Polvorosa, J. C; J, Bastiaensen (2016). Ganadería lechera y deforestación en Nicaragua. Encuentro, (104), 6-28.

Soto, R.; Lamela, L.; Sánchez, C. 2008. Producción de leche con una asociación de árboles Forrajeros y CT-115 bajo condiciones de riego. Universidad de Matanzas —Camilo Cienfuegos\| Estación Experimental de Pastos y Forrajes —Indio Hatueyll. 74 pp.

Torres, J. 2007. Validación de la gramínea forrajera brachiaria brizantha ciat 26110 en diferentes ambientes de Matagalpa y Jinotega, Nicaragua. Centro Experimental del Valle de Sébaco (CEVAS, /INTA Centro Norte., Matagalpa, Nicaragua. 110 pp.

Vilaboa-Arroniz, J., Díaz-Rivera, P., Ruiz-Rosado, O., Platas-Rosado, D. E., González-Muñoz, S., \& Juárez-Lagunes, F. (2009). Caracterización socioeconómica y tecnológica de los agroecosistemas con bovinos de doble propósito de la región del Papaloapan, Veracruz, México. Tropical and Subtropical Agroecosystems, 10(1).

Villanueva, C.; Ibrahim, M.; Casasola, F.; Ríos, N.; Sepúlveda, C. 2009. Políticas y sistemas de incentivos para el fomento y adopción de buenas prácticas agrícolas como una medida de adaptación al cambio climático en américa central. Sistemas silvopastoriles: una herramienta para la adaptación al cambio climático de las fincas ganaderas en América Central. Centro Agronómico Tropical de Investigación y Enseñanza. Claudia J. Sepúlveda L. y Muhammad Ibrahim. 103 - 125 p. 